\title{
MULTIDIMENSIONAL EXTENSIONS OF PÓLYA-KNOPP-TYPE INEQUALITIES OVER SPHERICAL CONES
}

\author{
Chang-Pao Chen, Jin-Wen LAn And DAH-Chin LuOR
}

\begin{abstract}
In this paper, we introduce a new type of limit process to evaluate the modular-type operator norm of an integral operator. This leads us to get multidimensional extensions of PólyaKnopp-type inequalities with general measures. Our results not only extend Levin-Cochran-Leetype inequalities from $n=1$ to general $n$, but also improve the estimates given there. Moreover, they generalize Carleson's result, which is involved in the proof of Carleman's inequality. Besides these, the Pólya-Knopp-type inequalities for the cases of Laplace transform and generalized Riemann-Liouville operators are derived. For the lower bounds, a parallel theory to the above is also established.
\end{abstract}

Mathematics subject classification (2010): 47A30, 26D10, 26D15.

Keywords and phrases: Operator norm, integral operator, multidimensional modular inequalities, HardyKnopp-type inequalities, Pólya-Knopp-type inequalities.

\section{REFERENCES}

[1] K. F. Andersen, H. P. Heinig, Weighted norm inequalities for certain integral operators, Siam J. Math. Anal. 14, no. 4, 834-844 (1983).

[2] L. CARleson, A proof of an inequality of Carleman, Proc. Amer. Math. Soc. 5, 932-933 (1954).

[3] C.-P. Chen, J.-W. LAN, D.-C. LuOR, The best constants for multidimensional modular inequalities over spherical cones, Linear and Multilinear Algebra, 62, Issue 5, pp. 683-713 (2014). DOI:10.1080/03081087.2013.777438.

[4] M. Christ, L. Grafakos Best constants for two nonconvolution inequalities, Proc. Amer. Math. Soc. 123, no. 6, 1687-1693 (1995).

[5] A. ČIŽMEŠIJA, J. PEČARIĆ, Some new generalisations of inequalities of Hardy and Levin-CochranLee, Bull. Austral. Math. Soc. 63, 105-113 (2001).

[6] A. ČIŽMEŠIJA, J. PEČARIĆ, I. PERIĆ, Mixed means and inequalities of Hardy and Levin-CochranLee type for multidimensional balls, Proc. Amer. Math. Soc., 128, no. 9, 2543-2552 (2000).

[7] A. ČIŽmešIJa, J. PeČArić, L.-E. Persson, On strengthened Hardy and Pólya-Knopp's inequalities, J. Approx. Theory 125, 74-84 (2003).

[8] J. A. Cochran, C.-S. LeE, Inequalities related to Hardy's and Heinig's, Math. Proc. Cambridge Philos. Soc. 96, 1-7 (1984).

[9] P. DrábeK, H. P. Heinig, A. Kunfer, Higher dimensional Hardy inequality, Intenat. Ser. Numer. Math. 123, 3-16 (1997).

[10] B. Gupta, P. Jain, L. E. Persson, A. Wedestig, Weighted geometric mean inequalities over cones in $\mathbb{R}^{N}$, J. Inequal. Pure Appl. Math. 4, Issue 4, Article 68 (2003).

[11] G. H. Hardy, J. E. Littlewood, G. Pólya, Inequalities, 2nd edition, Cambridge University Press, Cambridge (1967).

[12] H. P. HeINIG, Weighted norm inequalities for certain integral operators II, Proc. Amer. Math. Soc. 95, no. 3, 387-395 (1985).

[13] P. Jain, L.-E. Persson, A. Wedestig, Carleman-Knopp type inequalities via Hardy inequalities, Math. Inequal. Appl. 4(3), 343-355 (2001).

[14] A. Kufner, L.-E. Persson, Weighted inequalities of Hardy type, World Scientific Publishing Co., Singapore, New Jersey, London, Hong Kong (2003). 
[15] V. I. Levin, S. B. StečKIn, Inequalities, Amer. Math. Soc. Transl. (2), 14, 1-29 (1960).

[16] N. Levinson, Generalizations of an inequality of Hardy, Duke Math. J. 31, 389-394 (1964).

[17] E. R. Love Inequalities related to those of Hardy and of Cochran and Lee, Math. Proc. Cambridge Philos. Soc. 99, 395-408 (1986).

[18] V. M. ManaKov, On the best constant in weighted inequalities for Riemann-Liouville integrals, Bull. London Math. Soc. 24, 442-448 (1992).

[19] M. Nassyrova, L.-E. Persson, V. D. Stepanov, On weighted inequalities with geometric mean operator generated by the Hardy-type integral transform, J. Inequal. Pure Appl. Math. 3, Issue 4, Article 48 (electronic)(2002).

[20] L.-E. PERSSON, V. D. StePANOv, Weighted integral inequalities with the geometric mean operator, J. Inequal. Appl. 7, No. 5, 727-746 (2002).

[21] D. V. Prokhorov Weighted Hardy's inequalities for negative indices, Publications Matematiques, 48, 423-443 (2004).

[22] G. Sinnamon, One-dimensional Hardy-type inequalities in many dimensions, Proc. Roy. Soc. Edinburgh, 128A, 833-848 (1998).

[23] A. Wedestig, Weighted Inequalities of Hardy-type and their Limiting Iinequalities, $\mathrm{PhD}$ thesis 2003:17, Luleå University of Technology, Luleå 2003.

[24] R. L. Wheeden, A. Zygmund, Measure and Integral, Marcel Dekker Inc., New York (1977). 